Reseña de El americanismo germano en la antropología Argentina de fines del siglo XIX y XX de Lena Dávila y Patricia Arenas (Eds). Buenos Aires: Ediciones CICCUS- CLACSO, 2021. 523 páginas.

\title{
Marcos Buccellatto
}

\author{
(2) OpenEdition \\ Journals \\ Electronic version \\ URL: https://journals.openedition.org/corpusarchivos/5169 \\ DOI: 10.4000/corpusarchivos.5169 \\ ISSN: 1853-8037 \\ Publisher \\ Diego Escolar \\ Electronic reference \\ Marcos Buccellatto, «Reseña de El americanismo germano en la antropología Argentina de fines del siglo \\ XIX y XX de Lena Dávila y Patricia Arenas (Eds). Buenos Aires: Ediciones CICCUS- CLACSO, 2021. 523 \\ páginas. », Corpus [En línea], Vol. 11, №. 2 | 2021, Publicado el 16 diciembre 2021, consultado el 26 \\ diciembre 2021. URL: http://journals.openedition.org/corpusarchivos/5169 ; DOI: https://doi.org/ \\ 10.4000/corpusarchivos.5169
}

This text was automatically generated on 26 December 2021. 


\title{
Reseña de El americanismo germano en la antropología Argentina de fines del siglo XIX y XX de Lena Dávila y Patricia Arenas (Eds). Buenos Aires: Ediciones CICCUS- CLACSO, 2021. 523 páginas.
}

\author{
Marcos Buccellatto
}

1 La obra El americanismo Germano en la antropología argentina de fines del siglo XIX al siglo XX es un valioso esfuerzo colectivo de parte de un grupo de especialistas del área de la antropología y la historia, que se propone reponer las trayectorias de un conjunto de actores agrupados bajo el nombre de "americanistas germano-parlantes", en un periodo gestacional para la disciplina antropológica en Argentina y en Latinoamérica. Este apelativo reúne a naturalistas, aficionados y antropólogos de diferentes procedencias, diversa formación de base y distintos intereses y orientaciones, que no puede decirse que pertenezcan a una misma comunidad científica, pero que, sin embargo, desde una perspectiva histórica pueden integrarse en el relato de origen de la antropología argentina. El libro entonces conforma un doble aporte, por un lado a la historia de la antropología local y regional y, en términos más generales, a la historia de la ciencia argentina. De esta forma y como parte de la colección "Ciencia y Sociedad" dirigida por Cecilia Hidalgo de la editorial CICCUS, esta obra es también un espacio de debate y reflexión sobre la ciencia como indisolublemente ligada a lo social que debe ser entendida como empresa "contextualizada y colectiva".

2 El conjunto de trabajos que conforman el libro toman como eje el relato de vida de un personaje histórico, pero sin reducirse a la presentación de breves biografías. Al contrario, cada pieza aborda la vida de un actor iluminando las tensiones, los clivajes y problemas que atravesó la antropología en su proceso fundacional. Las trayectorias 
dispares y en apariencias inconexas son pinceladas que dan vida a un cuadro de una complejidad enorme que muestra las vinculaciones que existieron con otras áreas de la ciencia, múltiples procesos sociales e institucionales y diferentes momentos políticos y sociales del país. El desarrollo de los casos individuales permite entender mejor el punto de vista "nativo" propio de cada momento, las historias son contadas desde la perspectiva antropológica, pero con el cuidado y el rigor de la investigación histórica. De esta manera, toma lo mejor de los dos mundos disciplinarios.

El trabajo refleja un importante análisis de archivo y recopilación de información en un área poco trabajada en la literatura especializada, en particular en los casos de algunos personajes que son marginales o muy periféricos a los estereotipos, aparatos interpretativos y relatos canónicos sobre el origen de la antropología argentina. La pregunta que articula los diferentes trabajos que componen esta obra es: “¿Cómo dar cuenta de la emergencia de un campo de conocimiento cuando aún no existe un sistema de prácticas delimitado, un dominio de vida social separado, donde todavía no hay comunidades recortadas ni sitio o territorio científico privilegiado (por ejemplo, el laboratorio, la cátedra, entre otros?". Y como corolario de esto: ¿cómo hacerlo cuando los mismos actores involucrados no se autoperciben como parte del mismo colectivo, no están necesariamente en diálogo entre sí, y en muchos casos, no se cruzan más que de forma tangencial? La tarea no es fácil, por esto la interesante estrategia de las editoras, Lena Dávila y Patricia Arenas resulta en un conjunto de trabajos heterogéneos que permiten mostrar el papel de este grupo de "americanistas germano-parlantes" en el paisaje fundacional de la antropología local. Resulta valiosa también la estrategia de no establecer relaciones causales o relatos sobre héroes disciplinares, de no buscar un discurso unificador, sino uno que plantea un recorrido a través de la compleja y polifónica trama que va iluminándose a medida que se la transita. Así, los lectores se ven embarcados en un peregrinaje por distintas historias de vida abordadas desde diferentes perspectivas que van agregando nuevos matices al cuadro general. Al final de ese recorrido, el lector cosecha cierta familiaridad con el espíritu de cada momento histórico y las bases para comprender los vínculos y las relaciones con el posterior desarrollo de la disciplina. Quienes estén interesados en el estudio de la historia disciplinar local, encontrarán en estas páginas no una guía erudita, sino una experiencia que permite desenvolverse con comodidad para encarar futuras investigaciones.

4 Ahora bien, ¿por qué enfocar el grupo "germano" y no inglés? ¿o americano? ¿o francés? En primer lugar, como se menciona en el trabajo de Sergio Carrizo que inaugura la obra, había una intensa relación de intercambio científico entre Argentina y Alemania. Según los datos aportados, por ejemplo para el caso de los "naturalistas", un tercio de los investigadores de la Academia Nacional de Ciencias de Córdoba eran de origen alemán. Por otro lado, existía en el país una organización reticular de asistencia germana que servía de vínculo con Alemania, y que permitió a los extranjeros que venían a estas tierras posicionarse en espacios favorables. En el caso de la ciencia, esto se veía apalancado por el aura de prestigio que disfrutaban los académicos alemanes y europeos en general. Es interesante estudiar el rol de los extranjeros en la conformación de la ciencia local desde los trabajos que se presentan en el libro, desde las redes de vinculación, los roles en las instituciones, las relaciones con la producción científica local y el público para el que escribían y hacían ciencia. La actitud distante y menospreciativa que los académicos germanos a veces tenían para con sus pares 
argentinos, por ejemplo en los casos de Brumeister o Lehmann-Nitsche, y al mismo tiempo la mirada de admiración con que los locales veían a los científicos alemanes.

5 Sin embargo, esto último debe complejizarse en relación con los relatos individuales. Los germanos encontraban en la Argentina un espacio de desarrollo que no tenían en su tierra natal, y las instituciones locales, al mismo tiempo, se servían del capital simbólico que traía aparejado sumar extranjeros a sus filas y cargos jerárquicos. Así, la participación en esta red de intercambio tenía agendas diferentes para unos y para otros. Por último, considero que a la luz de la historia disciplinar local, el papel de la Escuela Alemana justifica la concentración de la atención en los germanos frente a otros grupos de actores. En este sentido, el papel de la Escuela Histórico Cultural en las obras de Bórmida, Menghin (tratado en uno de los trabajos) e Imbelloni entre otros y su papel en la conformación institucional de la antropología local, nos lleva a preguntarnos sobre qué tipo de vínculo previo había entre la academia alemana y la argentina. El trabajo de Rolando Silla que cierra este libro establece este puente de forma interesante, a través de la mirada de Márquez Miranda sobre la obra de Fritz Graebner.

6 La obra está construida en base a 16 artículos repartidos en cinco secciones temáticas. Las primeras dos partes tratan sobre grupos de actores que no podríamos identificar como antropólogos: son los "naturalistas", los "aficionados" y "coleccionistas". Esta inclusión ejemplifica una de las virtudes de la obra, que es abordar el proceso formativo de la ciencia antropológica local, cuando no hay un criterio de delimitación claro de lo que está dentro y fuera, y que la red de vinculaciones entre actores muy dispares va dando forma embrionaria a lo que luego será la disciplina establecida.

7 Los "naturalistas", aquellos "agentes sociales que establecieron criterios para definir órdenes genéricos, ontogénicos y morfológicos del mundo animal, vegetal y humano", aquellos con las mirada "objetiva" y descriptiva, incluyen a una serie de personajes muy diversos que comprende a académicos, exploradores y viajeros. Hay cuatro trabajos que caracterizan a este grupo, todos ellos ubicados en la segunda mitad del siglo XIX. El primer trabajo, de Sergio Carrizo, tiene su eje en la Academia Nacional de Ciencias de Córdoba y el rol que los académicos germanos tuvieron en ese lugar. Al final del mismo encontramos las observaciones y comentarios de Ezequiel Grisendi. El segundo, escrito por Pablo Perazzi y comentado por Christophe Giudicelli, versa sobre la trayectoria de Herman Burmeister y el museo público de Buenos Aires, una figura muy trascendente en la historia de la ciencia local que tuvo sus contrapuntos con la próxima generación de naturalistas locales que incluyen a Perito Moreno, Ameguino y Ambrosetti. El siguiente trabajo, de Daniel Delfino y Gustavo Pisani, sigue la historia de Paul Lorentz y la relaciona con el proyecto de organización nacional de fin de siglo. Un interesante trabajo que muestra las relaciones entre criollos e indios y expone el ideal de naturaleza que el nuevo proyecto nacional impone sobre el territorio y sus habitantes. En este caso encontramos los comentarios de Mariela Zabala. Por último, se presenta un trabajo sobre la trayectoria de Santiago Roth, relatada por Viviana Scheinsohn, donde se caracteriza la red suiza de asistencia y las vinculaciones con el Museo de la Plata y está comentado por Hugo Enrique Ratier.

8 La siguiente sección, la de los "aficionados y coleccionistas", también contiene cuatro artículos. El primero, escrito por Patricia Arenas, versa sobre la obra de Félix Methfessel, un artista y paisajista cuyos trabajos van a incluir desde representaciones de la guerra con el Paraguay hasta los dibujos y retratos de piezas pertenecientes al 
museo de Buenos Aires. Este artículo tiene comentarios de Pablo Tasso. Le sigue el capítulo, en coautoría de Viviana Scheinsohn y Nora Kuperzmit, sobre Wilhelm Vallentin y la colonia Friedland, ese lugar donde aún hoy se sigue diciendo "Acá no hubo indios", pese a las huellas innegables de su presencia histórica y presente. En este caso los comentarios estuvieron a cargo de Adriana Alejandra Stagnaro. El trabajo que le sigue, que pertenece a Axel Lazzari y Regula Nigg, trata sobre el médico alemán Luis Ruez, y es un mosaico de escenas de vida que pone en relación diversos eventos con temas como la red alemana local de circulación de conocimiento, o los vínculos con figuras académicas, las publicaciones, la vida rural, los indígenas y el límite entre el etnógrafo profesional y el aficionado. El artículo contó con los comentarios de Francine Masiello. Por último, es el trabajo de María Cristina Scattolin, sobre Vladimiro Weiser, un ingeniero responsable de una serie de expediciones al noroeste argentino y la formación de una gran colección de piezas y materiales. En sus minuciosas notas nunca publicadas se encuentran detalles que permiten reconstruir el pasado prehispánico. El tema de las colecciones, la circulación de piezas y el rol de los museos son temas omnipresentes en estas dos primeras secciones. Los comentarios finales corresponden a Barbara Manasse.

En tercer lugar, encontramos una sección con solo dos artículos, pero que tienen mucha importancia para entender la obra en su conjunto. El primero, de Barbara Göbel y comentado por Marisa Malvestitti, relata cómo era la circulación de objetos y el conocimiento entre Argentina y Europa, siguiendo las prácticas de Robert LehamnNitsche en el Museo de La Plata. Esta situación le deja el lugar preparado a un trabajo de índole más teórico, perteneciente a Cecilia Hidalgo con comentarios de Claudia Natenzon, que termina de dar forma al tema de las redes de conocimiento y sienta el tono con el que debe leerse el libro. Este artículo explicita el rol de las redes en el contexto de la actividad científica, los recursos que circulan, las ideas predominantes, las estrategias de posicionamiento de los actores, y los vínculos institucionales.

La cuarta sección, también compuesta por dos artículos, aborda un tema complicado y difícil de analizar sin caer en valoraciones anacrónicas: los posicionamientos políticoideológicos. Siguiendo las trayectorias de Robert Lehamn-Nietsche, bajo la pluma de Lena Dávila y los comentarios de Christine Lauriere, y Oswald Menghin, con la autoría de Patricia Arenas y comentardo por Pablo Andrés Castagno, dos polémicos personajes de la historia antropológica local, se analizan las respuestas y actitudes tomadas frente al nazismo. En el primer caso, en los momentos previos a la segunda guerra mundial y en el segundo en la post-guerra. Si bien los relatos giran en torno a dos personajes, permiten caracterizar el espíritu y la atmósfera intelectual de la época que marca el fin de la antropología alemana como tradición central. El rol de los intelectuales, sus posicionamientos políticos, sus silencios y omisiones son claves para entender este declive, y verlo desde la perspectiva local le da un valor agregado.

11 Por último nos encontramos con una sección dedicada a la recepción del americanismo germano y los vínculos intelectuales entre Alemania y Argentina. Abre el repertorio un trabajo de Alejandra Mailhe que explora la recepción de la obra de Oswald Spengler en base a la crítica que hace de ella Ernesto Quesada, los comentarios, en este caso, estuvieron a cargo de Ana Teresa Martinez. Le sigue un muy interesante artículo sobre la obra de Juliane Dillenius, la única antropóloga abordada en el libro y una figura pionera en la disciplina, no sólo en Argentina sino en Latinoamérica, con una importante trascendencia. El artículo refleja la importancia y el valor de sus aportes a 
la ciencia y las dificultades propias de intervenir en un campo escénicamente masculino en esa época. Este artículo corresponde a Paola Silvia Ramundo y los comentarios a Ana Carolina Arias. El anteúltimo trabajo, escrito por Javier Nastri y comentado por Per Cornell, sigue la historia de vida de Max Uhle, un personaje que no sólo fue influyente en el espacio local, sino que tuvo un rol importante en varios países latinoamericanos. Por último, y cerrando la obra, nos encontramos con el trabajo de Rolando Silla que discute la obra de Fritz Graebner, representante de la Escuela Histórico Cultural. Si bien Graebner no formó parte de la comunidad científica local, sus trabajos son importantes para poder entender a otros antropólogos como Bórmida e Imbelloni. Pese a esto, y manteniendo el foco local que tienen todos los trabajos, esta discusión se lleva adelante contraponiéndola con la mirada de Marquez Miranda, un abogado local adepto a la Escuela Histórico Cultural, pero con algunas críticas importantes a la misma. En este caso los comentarios los realizó Gastón Julián Gil.

Independientemente de la separación en diferentes secciones, los artículos dialogan muy bien entre ellos y muchas veces aportan diferentes perspectivas sobre los mismos temas complementando y enriqueciendo el análisis. Resulta muy interesante también que se haya decidido incluir al final de cada trabajo un comentario o crítica de otro especialista. Este diálogo y relectura, en la mayoría de los casos, aportan elementos nuevos de análisis. Este intercambio le da al libro una profundidad mayor, aunque lamentablemente, en algunos casos, los comentaristas simplemente se limitan a realizar un resumen de las ideas ya expresadas sin agregar mucho a la discusión. Pese a ello, en líneas generales los aportes son valiosos y enriquecedores. Resulta admirable el esfuerzo realizado por las editoras por coordinar los trabajos de más de 32 autores y colaboradores de todo el país y el extranjero para tener una mirada más amplia, federal y diversa.

13 Como se discute en el prólogo y en el capítulo que trata sobre las redes de producción de conocimiento, el libro más allá de brindar un aporte histórico invaluable, permite analizar el surgimiento de la antropología desde la perspectiva de los estudios sociales de la ciencia. Para quienes buscan trabajar en estas áreas, la casuística y los datos aportados son invaluables ya que abren el campo de discusión a múltiples variables y perspectivas que actúan en conjunto. Muestra también la dimensión humana del quehacer científico y las contingencias históricas que dieron forma a la disciplina.

\section{AUTHOR}

\section{MARCOS BUCCELLATTO}

Universidad Nacional de San Martín, Argentina. Correo electrónico: mbuccellato@unsam.edu.ar 